\title{
Ocorrência de fungos micorrízicos arbusculares em áreas revegetadas após mineração de bauxita em Porto Trombetas, Pará(1)
}

\author{
Ana Lucy Caproni(2), Avílio Antônio Franco ${ }^{(3)}$, Ricardo Luis Louro Berbara( ${ }^{(2)}$, Sandra Botelho Trufem ${ }^{(4)}$, \\ José Rodolfo Dantas de Oliveira Granha ${ }^{(2)}$ e Ana Beatriz Monteiro ${ }^{(5)}$
}

\begin{abstract}
Resumo - O objetivo deste trabalho foi avaliar a composição e a diversidade de fungos micorrízicos arbusculares (FMA) em áreas em processo de revegetação na região de Porto Trombetas, PA. Coletaram-se amostras compostas de solo em revegetação com 2, 4, 6, 12 e 16 anos, em subsolo exposto sem vegetação e em floresta primária, nos meses de agosto de 1998 (estação seca) e abril de 1999 (estação chuvosa). Os esporos de FMA foram extraídos e identificados taxonomicamente. Analisou-se a densidade relativa, a frequiência de cada espécie e os índices de Shannon-Wiener e de Simpson. Os resultados indicaram que a densidade de esporos de FMA não diferiu significativamente em razão da estação do ano. O número de espécies foi maior no período seco, na floresta primária e na área revegetada aos 2 anos de idade. Entre as espécies de FMA recuperadas, Glomus macrocarpum e Acaulospora mellea foram as que apresentaram maior ocorrência. A diversidade de espécies de FMA foi mais alta e a dominância de espécies foi mais baixa na área revegetada com 2 anos de idade. O retorno do horizonte superficial orgânico com o plantio de mudas micorrizadas é uma prática eficiente para a produção de esporos em subsolo resultante da mineração de bauxita.
\end{abstract}

Termos para indexação: micorriza vesicular-arbuscular, esporo, revegetação, recuperação ambiental.

\section{Arbuscular mycorrhizal fungi occurrence in revegetated areas after bauxite mining at Porto Trombetas, Pará State, Brazil}

\begin{abstract}
The objective of this study was to evaluate the structure and diversity of arbuscular mycorrhizal fungi (AMF) in revegetated areas at Porto Trombetas, Pará, Brazil. Composite samples were collected in August (dry season) and April (wet season) in areas with top soil after 2, 4, 6, 12 and 16 years of revegetation, in area without the top soil or vegetation, and in a pristine forest. The AMF spores were extracted, and the species identified. The relative density and frequency of occurrence of each species, the Shannon-Wiener and Simpson indexes were calculated. The results indicated that the density of AMF spores did not differ significantly with season. However, the number of AMF species was significantly higher in the dry season at the pristine forest and in the area two years after revegetation. Among the AMF species, Glomus macrocarpum and Acaulospora mellea were represented in higher numbers. After two years of planting, the diversity was highest and the dominance of AMF species was lowest. The addition of top soil with the planting of mycorrhizal seedlings were efficient to colonize areas with AMF after open cast bauxite mining.
\end{abstract}

Index terms: vesicular arbuscular mycorrhizae, spores, revegetation, environmental recovery.

(1) Aceito para publicação em 27 de outubro de 2003.

(2) Universidade Federal Rural do Rio de Janeiro, Dep. de Solos, Rodovia Rio-São Paulo, km 47, CEP 23890-970 Seropédica, RJ. E-mail: alcaproni2002@hotmail.com, berbara@ufrrj.br, rodolfogranha@yahoo.com.br

(3) Embrapa-Centro Nacional de Pesquisa de Agrobiologia, Caixa Postal 74505, CEP 23890-000 Seropédica, RJ. E-mail: avilio@cnpab.embrapa.br

(4) Universidade São Marcos, Unidade Central, Av. Nazaré, 900, CEP 04262-100 São Paulo, SP

E-mail: strufem@cyberspace.com.br

(5) Universidade Federal Fluminense, Dep. de Matemática, Rua Mário Santos Braga, s/no, Centro, CEP 24020-110 Niterói, RJ. E-mail: getabsn@vm.uff.br

\section{Introdução}

A mineração de bauxita no Estado do Pará destacase economicamente, apesar de causar grande impacto ambiental decorrente da remoção da cobertura vegetal e das camadas superficiais do solo. Uma das estratégias de recuperação dessas áreas é a reposição do horizonte superficial orgânico sobre o subsolo exposto da área minerada, o que favorece a disponibilidade de nutrientes para o restabelecimento de plantas e microrganismos, acelerando assim o processo de recupera- 
ção do solo (Pfleger et al., 1994). Os fungos micorrízicos arbusculares (FMA) auxiliam nos processos de revegetação, ao beneficiar o estabelecimento das mudas no campo, contribuindo para a absorção de nutrientes e água, além de atuar na proteção contra patógenos radiculares (Newsham et al., 1995).

Ao estudar os FMA em uma área minerada e revegetada, Gould et al. (1996) observaram que a densidade de propágulos de FMA foi baixa e não houve colonização das raízes das plantas introduzidas. Entretanto, no segundo ano, a densidade dos esporos e outros propágulos aumentou, estabilizando-se nos anos seguintes com a sucessão vegetal. Normalmente, os eventos registrados na população de FMA, nos primeiros 3 a 4 anos depois do plantio, são acompanhados por drásticas mudanças na vegetação ainda com a diversidade de FMA mostrando correlações positivas com a sucessão vegetal (Pfleger et al., 1994; Gould \& Hendrix, 1998).

Uma forma de estudar as comunidades de FMA é expressar a diversidade de espécies de um ecossistema pelo cálculo de índices (Odum, 1988). A diversidade é resultante da riqueza de espécies, também chamada de densidade ou abundância de espécies, que é baseada no número total de espécies presentes, e da uniformidade, também chamada de eqüitabilidade, que expressa a abundância relativa de espécies assim como o grau de dominância de uma espécie em relação a outras (Odum, 1988; Kennedy \& Smith, 1995). Os índices de diversidade, que avaliam a riqueza e dominância, mais usados são o de Shannon-Wiener e o de dominância de Simpson (Pielou, 1983).

Existem limitações ao uso de índices de diversidade, os quais podem assumir valores extremos, quando usados na caracterização de comunidades de FMA (Franke-Snyder et al, 2001). Contribuem para isto a limitação, causada pelo uso dos esporos, na identificação dos FMA (Morton et al., 1995) e o fato destes, muitas vezes, não refletirem a estrutura da comunidade vegetativa (Douds \& Millner, 1999). Entretanto, como os índices de diversidade são usados para avaliação das comunidades de um mesmo ambiente, avaliações das populações de FMA poderão indicar os padrões de esporulação destes fungos associados à sucessão florestal, quando comparados com uma floresta primária.
O objetivo deste trabalho foi avaliar a composição e a diversidade das comunidades de FMA em áreas em processo de revegetação, depois da mineração de bauxita em Porto Trombetas, PA.

\section{Material e Métodos}

O Distrito de Porto Trombetas $\left(56^{\circ} \mathrm{W}, 1^{\circ} 40^{\prime} \mathrm{S}\right)$, no Município de Oriximiná, está localizado na região oeste do Pará, 100 km a Oeste da confluência do Rio Trombetas com o Rio Amazonas. De acordo com a classificação de Köppen, o clima regional é do tipo $\mathrm{AW}$, bem definido, com estação seca e chuvosa (Ferraz, 1993). Na região ocorre uma associação de argissolos profundos, que apresentam um horizonte B, de textura argilosa, acidez, além de serem plásticos e pegajosos. São solos de baixa fertilidade natural, baixa capacidade de troca de cátions e baixos níveis de saturação por bases (Ferraz, 1993).

A recuperação das áreas com histórico de mineração teve início com a distribuição de uma camada de horizonte superficial orgânico, de aproximadamente $20 \mathrm{~cm}$ de espessura, sobre o subsolo de aproximadamente oito metros de profundidade, exposto após a extração de bauxita. A revegetação foi feita com o plantio de mudas de aproximadamente 100 espécies arbóreas nativas, incluindo, dependendo do ano, até quatro espécie exóticas (Salomão et al., 1997). Na produção de mudas, foram usadas sementes, coletadas na própria região, de espécies dos gêneros Ambelania, Anacardium, Andira, Aniba, Annona, Apeiba, Bowdichia, Brosimum, Caryocar, Cassia, Copaifera, Couma, Dalbergia, Dimorphandra, Diplotropis, Endopleura, Enterolobium, Eshweilera, Goupia, Guatteria, Hymeneae, Hymenolobium, Inga, Iryanthera, Jacaranda, Lacmellea, Laetia, Lecythis, Leucaena, Licania, Luhea, Naucleopsis, Ocotea, Oenocarpus, Peltogyne, Platymiscium, Protium, Psidium, Sacoglotis, Sclerolobium, Senna, Sesbania, Spondias, Stryphnodendron, Swartzia, Tachigali, Tapirira, Taralea, Trattinickia, Vataira, Vismia. O substrato empregado foi o horizonte superficial orgânico da floresta primária (Lapa, 2000), o qual continha inóculos naturalmente provenientes do campo.

\section{Amostragens}

Para monitorar a ocorrência de FMA, foram coletadas amostras de solos em áreas com reposição do horizonte superficial orgânico e revegetadas, aos 2, 4, 6 e 12 anos após o plantio; em área de subsolo exposto, denominado estéril (um ano após a mineração e não revegetada); em uma área revegetada há 16 anos, diretamente sobre o subsolo estéril e em uma área de floresta primária. Foram 
coletadas, aleatoriamente, quatro amostras compostas, constituídas de 10 subamostras, na estação seca (agosto/1998) e chuvosa (abril/1999), numa área aproximada de $100 \mathrm{~m}^{2}$. As subamostras foram coletadas na profundidade de $0-20 \mathrm{~cm}$, na região radicular da vegetação As subamostras foram homogeneizadas e as amostras compostas foram secadas à sombra, acondicionadas em sacolas de plástico e armazenadas a $10^{\circ} \mathrm{C}$, até o seu processamento em laboratório, para isolamento de esporos de FMA e encaminhamento para análise das características físicoquímicas (Tabela 1).

\section{Extração dos esporos e preparo das lâminas}

De cada amostra composta, retirou-se $100 \mathrm{~mL}$ de solo para as extrações dos esporos pela técnica de peneiramento em via úmida (Gerdemann \& Nicolson, 1963), seguindo-se centrifugação com sacarose $50 \%$. Após a contagem, os esporos foram transferidos para uma placa de Petri e agrupados pelo tamanho, cor e forma. A seguir, foram colocados em lâminas com álcool polivinil em lactoglicerol (PVLG) e quebrados sob lamínula, a fim de expor as paredes internas. Na mesma lâmina, um segundo grupo de esporos foi montado com PVLG + reagente de Melzer (1:1), sob outra lamínula. Os resultados da reação de cor ao reagente de Melzer foram usadas para caracterizar as paredes dos esporos, melhorando, em alguns casos, a visibilidade, especialmente daqueles com paredes aderentes ou muitos finas. Os esporos foram então identificados e contados por espécie.

\section{Identificação das espécies de FMA}

A identificação das espécies de FMA foi feita segundo Schenck \& Perez (1988) e conforme descrição morfológica disponível na internet, na página da International Culture Collection of Arbuscular Mycorrhizal Fungi (West Virginia University, 2000), mediante observações em microscópio óptico com iluminação de campo-claro e objetiva de imersão. Os esporos foram contados, mesmo quando parasitados ou não viáveis.

\section{Análise dos dados}

Estimou-se a densidade de FMA (D) por meio do número de esporos em $100 \mathrm{~mL}$ de solo e a densidade de cada espécie de FMA $\left(\mathrm{D}_{\mathrm{i}}\right)$, como sendo a relação entre o número de esporos de determinada espécie por $100 \mathrm{~mL}$ de solo. As diferenças nas densidades entre o número total de esporos e o de espécies, entre as épocas de amostragens e as áreas amostradas, foram analisadas pelo teste nãoparamétrico de Mann-Whitney (Noether, 1983). A freqüência de cada espécie $\left(F_{i}\right)$ foi calculada em cada época de amostragens (seca e chuvosa) (Brower et al., 1990), de acordo com a equação $F_{i}=J_{i} / K$, em que $F_{i}$ é a frequiência da espécie $\mathrm{i}$; $\mathrm{J}_{\mathrm{i}}$ é o número de amostras nos quais a espécie i ocorreu e K é o número total de amostras de solo.

A frequiência relativa de cada espécie de $\mathrm{FMA}\left(\mathrm{FR}_{\mathrm{i}}\right)$ foi estimada, para o período total que incluiu as duas épocas de amostragens (seca + chuvosa), como a proporção da soma das freqüências para todas as espécies (Brower et al., 1990), representada pela equação $F R_{i}=f_{i} / \sum \mathrm{f}$, em que $F R_{i}$ é a frequiência relativa de uma dada espécie $\mathrm{i} ; \mathrm{f}_{\mathrm{i}}$ é a freqüência de uma dada espécie i; $\sum$ fé a soma das freqüências de todas as espécies.

Os índices de riqueza de espécies Shannon-Wiener e de dominância de Simpson foram calculados para análise das comunidades (Hendrix et al., 1995) em cada amostra de solo. A riqueza de espécies foi determinada como uma relação entre o número de espécies observadas e o tamanho da amostra (100 $\mathrm{mL}$ de solo). O índice de diversidade de Shannon-Wiener (H') foi calculado para cada amostra, de acordo com a equação:

$\mathrm{H}^{\prime}=-\Sigma\left(\mathrm{X}_{\mathrm{i}} / \mathrm{X}_{\mathrm{o}}\right) \times \log \left(\mathrm{X}_{\mathrm{i}} / \mathrm{X}_{\mathrm{o}}\right)$,

em que $X_{i}$ é a densidade de esporos de cada espécie em $100 \mathrm{~mL}$ de solo; $\mathrm{X}_{\mathrm{o}}$ é a densidade total de esporos de todas as espécies.

O índice de dominância de Simpson (C) foi calculado pela equação $\mathrm{C}=\Sigma\left(\mathrm{X}_{\mathrm{i}} / \mathrm{X}_{\mathrm{o}}\right)^{2}$. Os índices foram calculados para cada amostra de solo e submetidos à análise de variância. Foi usado o teste de médias para comparar os índices nas áreas e épocas de avaliação.

Tabela 1. Análise química das amostras de solos das áreas revegetadas após a mineração de bauxita, coletadas no mês de agosto de 1998.

\begin{tabular}{|c|c|c|c|c|c|c|c|c|}
\hline Áreas & $\mathrm{N}$ & $\mathrm{C}$ orgânico & $\mathrm{pH}$ & $\mathrm{Al}^{3+}$ & $\mathrm{Ca}^{2+}$ & $\mathrm{Mg}^{2+}$ & $\mathrm{P}$ & $\mathrm{K}$ \\
\hline & & $\mathrm{g} / \mathrm{dm}$ & & & $\frac{1 \mathrm{c} / 100}{(1)}$ & & 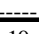 & \\
\hline Subsolo exposto (sem plantio) & 0,03 & 0,12 & 4,4 & 0,2 & $-(1)$ & - & 10 & 48 \\
\hline Subsolo exposto reflorestado com 16 anos & 0,19 & 1,77 & 4,2 & 0,7 & 1,3 & 0,6 & 0 & 35 \\
\hline Floresta primária & 0,14 & 1,08 & 4,0 & 1,0 & - & - & 0 & 22 \\
\hline Revegetação com dois anos & 0,16 & 1,89 & 4,3 & 1,0 & - & - & 1 & 39 \\
\hline Revegetação com quatro anos & 0,14 & 1,62 & 4,1 & 0,9 & - & - & 0 & 29 \\
\hline Revegetação com seis anos & 0,15 & 1,86 & 3,6 & 1,8 & - & - & 1 & 22 \\
\hline Revegetacão com 12 anos & 0,14 & 1,53 & 4,0 & 1,3 & - & - & 1 & 17 \\
\hline
\end{tabular}

(1)Não detectado pela análise. 


\section{Resultados e Discussão} estações do ano tanto em densidade de esporos como em número de espécies, com a maior riqueza ocorrendo em períodos secos e maior densidade em períodos úmidos. A densidade de esporos diferiu significativamente, pelo teste Mann-Whitney $(p \leq 0,05)$, entre as duas épocas de coleta somente nas amostras provenientes do subsolo exposto revegetado há 16 anos (Figura 1). Este fato pode relacionar-se com a prevalência do clima local somente em uma área, onde a revegetação ocorreu sem a reposição do horizonte superficial orgânico. Nas demais áreas revegetadas, observou-se um aumento do número de esporos, com a sucessão até a sua estabilização, quando a densidade de FMA decresce, conforme

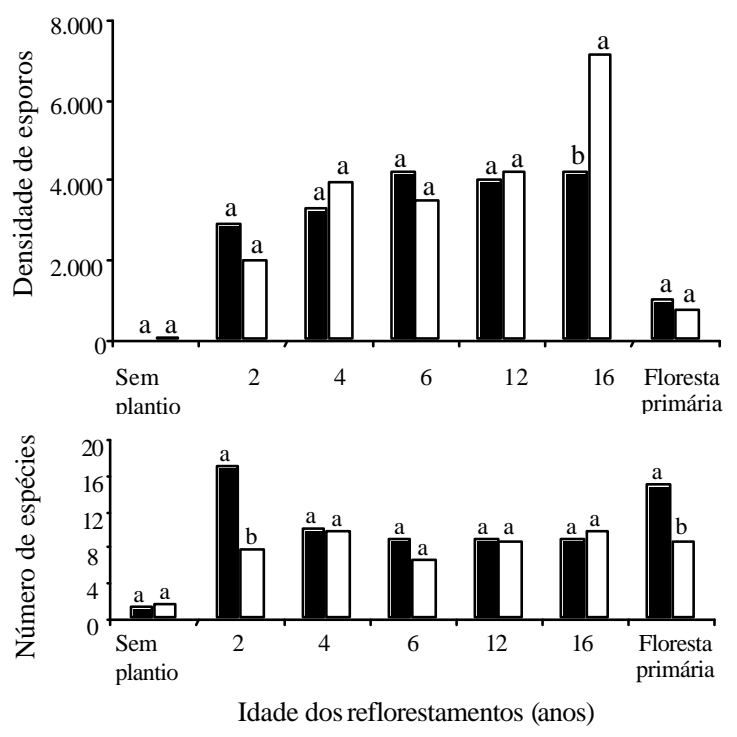

Teríodo seco ago./1998 प Período chuvoso abr./1999

Figura 1. Densidade de esporos de fungos micorrízicos arbusculares (FMA) e número de espécies de FMA. Amostras contendo $100 \mathrm{~mL}$ de solo na área de subsolo exposto sem plantio, nas áreas revegetadas com a reposição da camada superficial orgânica há 2, 4, 6 e 12 anos, na área com 16 anos de revegetação sem a reposição da camada superficial orgânica e na floresta primária. Colunas na mesma idade de revegetação com mesma letra não diferem entre si, nas duas épocas de amostragens, pelo teste de Mann-Witney, a 5\% de probabilidade.
As populações de FMA variaram pouco entre as

verificado pelos baixos valores encontrados na floresta primária.

Tanto a densidade de esporos quanto o número de espécies de FMA apresentaram valores significativamente menores na área não revegetada (subsolo estéril) do que nas demais áreas nas duas épocas de amostragens (Figura 1 e Tabela 2). O número de espécies de FMA das amostras de solo da floresta primária foi similar ao das amostras de solo da área revegetada há dois anos (Figura 1), com maior número de espécies na época seca em relação à chuvosa. A área revegetada há dois anos apresentou número de espécies de FMA significativamente maior do que as áreas com 4, 6, 12 e 16 anos de reflorestamento (Tabela 2) e não apresentou diferença significativa em relação à floresta primária $(\mathrm{p} \leq 0,05)$ no período seco.

No período chuvoso, a floresta primária não diferiu das demais áreas revegetadas quanto ao número de espécies de FMA. Quando houve efeito da época

Tabela 2. Níveis de significância, segundo teste nãoparamétrico de Mann-Whitney, em relação à densidade de esporos e número de espécies de fungos micorrízicos arbusculares nas coletas de amostras de solo, no período seco (ago./1998) e chuvoso (abr./1999), nas áreas revegetadas com reposição da camada superficial orgânica com 2, 4, 6 e 12 anos de idade; na área sem plantio (estéril); na área com 16 anos de revegetação sem a reposição da camada superficial orgânica e na floresta primária ${ }^{(1)}$.

\begin{tabular}{|c|c|c|c|c|}
\hline \multirow[t]{2}{*}{ Interação } & \multicolumn{2}{|c|}{ Número total de esporos } & \multicolumn{2}{|c|}{ Número de espécies } \\
\hline & Per. seco & Per. chuvoso & Per. seco & Per. chuvoso \\
\hline Estéril x 2 anos & * & * & * & * \\
\hline Estéril x 4 anos & * & $*$ & $*$ & $*$ \\
\hline Estéril x 6 anos & $*$ & $*$ & $*$ & $*$ \\
\hline Estéril x 12 anos & * & $*$ & * & * \\
\hline Estéril x 16 anos & $*$ & $*$ & $*$ & $*$ \\
\hline Estéril x FP & $*$ & $*$ & $*$ & $*$ \\
\hline 2 anos $\mathrm{x} 4$ anos & $\mathrm{ns}$ & $\mathrm{ns}$ & * & $\mathrm{ns}$ \\
\hline 2 anos $\times 6$ anos & $*$ & $*$ & * & $\mathrm{ns}$ \\
\hline 2 anos x 12 anos & ns & $*$ & $*$ & $\mathrm{~ns}$ \\
\hline 2 anos x 16 anos & $\mathrm{ns}$ & $*$ & $*$ & $\mathrm{~ns}$ \\
\hline 2 anos $x$ EP & $*$ & $*$ & ns & ns \\
\hline 4 anos $\times 6$ anos & $\mathrm{ns}$ & $\mathrm{ns}$ & $\mathrm{ns}$ & $*$ \\
\hline 4 anos x 12 anos & $\mathrm{ns}$ & $\mathrm{ns}$ & $\mathrm{ns}$ & $\mathrm{ns}$ \\
\hline 4 anos $x 16$ anos & $\mathrm{ns}$ & $*$ & $\mathrm{~ns}$ & $\mathrm{~ns}$ \\
\hline 4 anos x FP & $*$ & $*$ & $*$ & $\mathrm{~ns}$ \\
\hline 6 anos $x 12$ anos & $\mathrm{ns}$ & ns & $\mathrm{ns}$ & $\mathrm{ns}$ \\
\hline 6 anos $\times 16$ anos & $\mathrm{ns}$ & $*$ & $\mathrm{~ns}$ & $\mathrm{~ns}$ \\
\hline 6 anos x FP & $*$ & $*$ & $*$ & $\mathrm{~ns}$ \\
\hline 12 anos x 16 anos & $\mathrm{ns}$ & $*$ & $\mathrm{~ns}$ & $\mathrm{~ns}$ \\
\hline 12 anos $\times$ FP & $*$ & $*$ & $*$ & $\mathrm{~ns}$ \\
\hline 16 anos $x$ EP & $*$ & $*$ & $*$ & ns \\
\hline
\end{tabular}


na diversidade, ela foi maior no período seco, sugerindo que o estresse hídrico estimulou a esporulação de maior número de espécies. Gould et al. (1996) também observaram que a esporulação dos FMA foi parcialmente afetada pela estação do ano.

Pesquisadores têm observado que há maior variação na densidade de esporos de FMA entre o período seco e chuvoso somente em áreas com menor cobertura vegetal (Martins et al., 1999). Talvez isto explique a razão de, nas áreas estudadas, com cobertura intensa, não terem sido encontradas diferenças significativas, com exceção da área de 16 anos, onde o solo encontrava-se com baixa cobertura. Na área de subsolo exposto, a presença de menores densidades de esporos e números de espécies já era esperada, pois existe um declínio exponencial do número de esporos com a profundidade do solo, sendo encontrada maior densidade nos primeiros $20 \mathrm{~cm}$ de profundidade (Abbott \& Robson, 1991). Neste trabalho, o solo foi revolvido a uma profundidade de $13 \mathrm{~m}$, o que certamente teve efeito deletério sobre os microrganismos.

Em relação às áreas revegetadas, a floresta primária apresentou uma densidade de esporos, significativamente mais baixa (com valores entre $777 \mathrm{e}$ 1.040 esporos em $100 \mathrm{~mL}$ de solo). Munyanziz et al. (1997) observaram que a densidade de esporos de FMA é muito baixa em florestas não perturbadas, aumentando com baixo ou moderado grau de perturbação. Por outro lado, na floresta primária, encontrou-se menor densidade de esporos que na área reflorestada há dois anos. Isto é resultado da camada orgânica ter sido devolvida ao local de revegetação, servindo como fonte de inóculo. A grande quantidade de espécies de FMA encontradas na área revegetada há dois anos pode estar relacionada à introdução da camada superficial orgânica do solo antes do plantio. Após o segundo ano, a população de FMA declinou, voltando a se restabelecer com a idade do reflorestamento.

A menor densidade de esporos de FMA e maior riqueza de espécies, encontradas na floresta primária, pode ser conseqüência da sua maior estabilidade, menor competição por nichos (guildas), dando garantia da sobrevivência das espécies com baixa esporulação. Pode-se considerar, também, a presença de uma biota micófaga mais ativa ou a predomi- nância de espécies não pioneiras (espécies K), que não induzem grandes esporulações (Sieverding, 1991). As demais áreas (reflorestamento com 4, 6 e 12 anos de idade) apresentaram alta densidade média de esporos, variando entre 3.320 e 4.203 esporos em $100 \mathrm{~mL}$ de solo, fato indicador de que as espécies vegetais usadas no programa de revegetação induzem grande multiplicação inicial de esporos, possivelmente, por serem pioneiras e terem alta capacidade de adaptação (espécies R).

As áreas amostradas apresentaram um total de 57 espécies de FMA, pertencentes a seis gêneros, quatro famílias e duas subordens. Deste total, 53 espécies ocorreram nas amostras de solo coletadas na época seca (agosto) e 39 espécies na época chuvosa (abril), indicando uma maior diversidade em períodos de estresse hídrico. O maior número de espécies identificadas em todas as áreas amostradas pertenceu ao gênero Glomus (G. caledonium, G. claroideum, G. etunicatum, G. fasciculatum, G. formosanum, G. geosporum, G. glomerulatum, G. hoi, G. intrarradices, G. invernaium, G. macrocarpum, G. magnicaule, G. microcarpum, G. nanolumem, G. reticulatum, $G . \mathrm{sp} 1, G . \mathrm{sp} 2, G$. sp3, G. sp4, G. sp5, G. sp6, G. sp7, G. sp8, G. sp9, G. sp10, G. clavisporum e $G$. sinuosum), seguido pelo gênero Acaulospora (A. foveata, A. laevis, A. mellea, A. morrowiae, A. rehmi, A. scrobiculata, A. sp1, A. sp2, A. sp3, A. spinosa e A. tuberculata), Entrophospora (E. colombiana, E. contingua, E. kentinensis, E. sp1, E. sp2, E. sp3 e E. sp4), Scutellospora (S. calospora, S. gilmorei, $S$. gregaria, S. heterogama, S. sp1e $S$. weresubiae), Gigaspora (G. gigantea, G. margarita, G. rosea e G. sp1) e Archeospora (A. leptoticha), representando respectivamente $49 \%, 19 \%, 12 \%, 11 \%, 7 \%$ e $2 \%$ do total das espécies encontradas em todo o levantamento.

Foram encontradas 12 espécies de Acaulospora no período seco e nove no período chuvoso, com predomínio de A. mellea, numa freqüência que variou de $64 \%$ a $50 \%$ e com uma densidade relativa entre $5 \%$ e $2 \%$, respectivamente, nos períodos seco e úmido (Tabela 3). Todas as espécies deste gênero apresentaram diminuição da freqüência no período chuvoso. Algumas espécies de Acaulospora têm sido encontradas com alta freqüência nos trópicos. 
Tabela 3. Densidade relativa e frequiência das espécies de FMA presentes no período seco (ago./1998) e chuvoso (abr./ 1999), nas áreas revegetadas com reposição da camada superficial orgânica com 2, 4, 6 e 12 anos de idade, na área sem plantio, na área revegetada há 16 anos, sem a reposição da camada superficial orgânica e na floresta primária.

\begin{tabular}{|c|c|c|c|c|}
\hline \multirow[t]{2}{*}{ Espécies } & \multicolumn{2}{|c|}{ Densidade relativa } & \multicolumn{2}{|c|}{ Freqüência } \\
\hline & Período seco & Período chuvoso & Período seco & Período chuvoso \\
\hline Acaulospora foveata & 1,00 & 1,94 & 39 & 32 \\
\hline A. laevis & 1,32 & - & 14 & - \\
\hline A. mellea & 5,17 & 1,92 & 64 & 50 \\
\hline A. morrowiae & 0,32 & 0,60 & 29 & 21 \\
\hline A. rehmi & 0,04 & 0,32 & 14 & 11 \\
\hline A. scrobiculata & 1,19 & 0,11 & 25 & 4 \\
\hline A. spinosa & 0,20 & 0,04 & 7 & 4 \\
\hline A. tuberculata & 2,31 & 2,60 & 50 & 39 \\
\hline Acaulospora $\mathrm{sp} 1$ & 0,04 & 0,30 & 4 & 7 \\
\hline Acaulospora $\mathrm{sp} 2$ & 0,44 & - & 14 & - \\
\hline Acaulospora $\mathrm{sp} 3$ & 0,08 & - & 7 & - \\
\hline Archeospora leptoticha & 0,30 & 0,61 & 25 & 14 \\
\hline Entrophospora colombiana & 4,71 & 0,04 & 25 & 4 \\
\hline E. contingua & 1,03 & - & 7 & - \\
\hline E. kentinensis & 0,01 & - & 4 & - \\
\hline 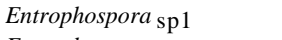 & 0,86 & 0,13 & 25 & 7 \\
\hline Entrophospora sp2 & 0,41 & - & 11 & - \\
\hline Entrophospora $\mathrm{sp} 3$ & 0,06 & - & 7 & - \\
\hline Entrophospora sp4 & 0,30 & - & 14 & - \\
\hline Gigaspora gigantea & 0,01 & 0,01 & 4 & 4 \\
\hline G. margarita & 0,30 & 0,16 & 25 & 14 \\
\hline G. rosea & 0,02 & - & 4 & - \\
\hline Gigaspora $\mathrm{sp1}$ & 0,05 & - & 11 & - \\
\hline Glomus caledonium & 0,06 & 0,07 & 7 & 4 \\
\hline G. claroideum & 1,16 & 2,30 & 18 & 21 \\
\hline G. clavisporum & - & 0,43 & - & 7 \\
\hline G. etunicatum & 3,83 & 2,40 & 61 & 57 \\
\hline G. fasciculatum & 0,24 & - & 14 & - \\
\hline G. formosanum & 0,28 & 1,23 & 25 & 36 \\
\hline G. geosporum & 0,65 & 0,88 & 18 & 25 \\
\hline G. glomerulatum & - & 0,43 & - & 7 \\
\hline G. hoi & 0,03 & - & 4 & - \\
\hline G. intrarradices & 0,67 & 0,12 & 14 & 11 \\
\hline G. invernaium & 0,11 & - & 11 & - \\
\hline G. macrocarpum & 52,12 & 63,62 & 93 & 93 \\
\hline G. magnicaule & 0,06 & 0,06 & 4 & 7 \\
\hline G. microagregatum & 0,01 & - & 4 & - \\
\hline G. microcarpum & 0,18 & 0,20 & 7 & 7 \\
\hline G. nanolumen & 0,04 & 3,85 & 7 & 32 \\
\hline G. reticulatum & 4,49 & 4,85 & 29 & 32 \\
\hline G. rubiformis & 0,39 & - & 21 & - \\
\hline G. sinuosum & - & 0,28 & - & 4 \\
\hline Glomus sp1 & 8,81 & 4,34 & 68 & 50 \\
\hline Glomus sp10 & 0,06 & - & 4 & - \\
\hline Glomus $\mathrm{sp} 2$ & 0,72 & 1,10 & 14 & 32 \\
\hline Glomus sp3 & 0,34 & 0,07 & 18 & 4 \\
\hline Glomus sp4 & - & 0,01 & - & 4 \\
\hline Glomus sp5 & - & 0,17 & - & 4 \\
\hline Glomus sp6 & 0,66 & 0,45 & 29 & 25 \\
\hline Glomus sp7 & 0,03 & 1,24 & 4 & 11 \\
\hline Glomus sp8 & 0,01 & - & 4 & - \\
\hline Scutellospora calospora & 2,75 & 1,93 & 46 & 36 \\
\hline S. gilmorei & 0,12 & - & 7 & - \\
\hline S. gregaria & 0,02 & - & 4 & - \\
\hline S. heterogama & 0,01 & 0,01 & 4 & 4 \\
\hline S. weresubiae & 0,06 & 0,24 & 4 & 11 \\
\hline Scutellospora $_{\mathrm{sp} 1}$ & 0,09 & 0,45 & 11 & 7 \\
\hline
\end{tabular}


Siqueira et al. (1989) encontraram A. morrowiae, em vários ecossistemas do Estado de Minas Gerais durante o verão, com elevada frequiência (acima de 90\%). Carrenho (1998) encontrou A. foveata com uma freqüência de $30 \%$ em um solo cultivado com milho. Foi relatada, também, em um solo florestal por Klironomos et al. (1993), com mais de $95 \%$ da população total dos esporos, sendo negativamente correlacionada com o $\mathrm{pH}$.

No período seco, foram observadas sete espécies do gênero Entrophospora e no período chuvoso, apenas duas espécies (Tabela 3). Esporos de Entrophospora colombiana apresentaram freqüência de $25 \%$ no período seco e $4 \%$ no período chuvoso. Sua densidade relativa variou de $5 \%$ (período seco) a $0,04 \%$ (período úmido). As demais espécies desse gênero foram encontradas apenas no período seco, com menos de $15 \%$ de ocorrência. E. colombiana tem sido relatada como de ocorrência equatorial e comum em solos perturbados e revegetados (Cuenca et al., 1998), e tem ampla distribuição nos ecossistemas brasileiros (Siqueira et al., 1989; Carrenho, 1998; Martins et al., 1999). Segundo Mehrotra (1998), esta espécie apresenta maior esporulação em solos ácidos que neutros ou alcalinos.

Das espécies do gêneroGigaspora, G. margarita apresentou maior frequiência, com $25 \%$ e $14 \%$, respectivamente, nos períodos seco e úmido (Tabela 3 ). Sua densidade relativa foi entretanto baixa, variando entre $0,3 \%$ e $0,16 \%$. No levantamento realizado, as espécies do gênero Gigaspora foram encontradas em baixa freqüência, apesar de citada por Clark (1997) como uma espécie comum de solos ácidos da América do Sul, tendo baixa esporulação em solos com pH 4,5 e alta com pH 5,5. As demais espécies apresentaram freqüência menor ou igual a $11 \%$. As espécies do gênero Gigaspora apresentaram baixa densidade relativa (abaixo de $0,3 \%$ ).

Foram encontradas, no período seco, 23 espécies de Glomus e no período chuvoso, 21 espécies (Tabela 3). Glomus macrocarpum e G. etunicatum apresentaram freqüência de esporos acima de $50 \%$, tanto no período seco quanto no período chuvoso. Glomus reticulatum e G. nanolumen apresentaram a mesma freqüência no período chuvoso e seco. Outras espécies aparecem no período chuvoso e não no seco, como G. glomerulatum, G. clavisporum e G. sinuosum. Glomus macrocarpumapresentou densidade relativa muito elevada, tanto no período chuvoso (64\%) como no seco (52\%). A frequiência também foi elevada, com $93 \%$ em ambos os períodos. A alta freqüência e alta densidade relativa das espécies do gênero Glomus, em especial Glomus macrocarpum, indicou sua maior capacidade de esporulação ou alta adaptabilidade à região e à situações iniciais de sucessão, independentemente das condições climáticas.

Esporos de G. macrocarpumtêm sido encontrados em áreas revegetadas após mineração de carvão com pH variando de 3,2 a 7,1 (Kierman et al., 1983) e em solos agrícolas com pH entre 4,5 e 5,5 (Clark, 1997; Santos et al., 2000), mostrando sua ampla faixa de adaptação. É importante conhecer mais detalhadamente a ecologia desta espécie de FMA, por apresentar potencial para o uso em programas de recuperação de áreas degradadas nos trópicos.

Dentro do gênero Scutellospora, a S. calospora apresentou maior freqüência e densidade relativa tanto no período seco quanto no período chuvoso (Tabela 3). Sua densidade variou de 2,7\% (período seco) a $1,9 \%$ (período úmido) enquanto sua freqüência no período seco foi de $46 \%$ e no úmido de $36 \%$. Scutellospora heterogama apresentou densidade relativa e frequiência mais baixa que as demais, mas foi detectada nos dois períodos de amostragens. Scutellospora gilmorei e $S$. gregaria ocorreram apenas no período seco, enquanto $S$. calospora, $S$. heterogama e $S$. weresubiae não demonstraram preferência de esporulação, ocorrendo em ambos os períodos com baixa frequiência e densidade. A falta de relação entre freqüência e abundância de esporos indica uma distribuição espacial desuniforme dos esporos por causa da presença de diferentes nichos no solo (Tews \& Koske, 1986).

A diversidade de espécies avaliada pelo índice de Shannon-Wiener revelou resultados diferentes nas áreas de plantio em sucessão (Figura 2). A área com dois anos apresentou alta diversidade, uma vez que a composição de sua população foi induzida pelo solo superficial coletado logo após a derrubada da floresta e reposto sobre o subsolo exposto. Esse manejo permitiu uma recomposição da população de FMA, a qual declinou e passou a aumentar com a 
sucessão vegetal. A diminuição da dominância de espécies de FMA com o aumento do tempo de revegetação, conforme indicada pelo índice de Simpson, mostrou uma tendência de melhor distribuição das populações em ecossistema clímax, conforme Allen (1991).

Na floresta primária, a dominância de espécies foi inferior a das áreas reflorestadas (Figura 2), sugerindo haver um aumento na eqüitabilidade entre espécies com a sucessão vegetal. Comparando-se o índice de diversidade de espécies de Shannon-Wiener com o índice de dominância de espécies de Simpson (Figura 2), observa-se que, onde a diversidade das espécies foi mais alta, a dominância foi mais baixa. A principal espécie responsável por esse efeito foi Glomus macrocarpum, que apresentou alta
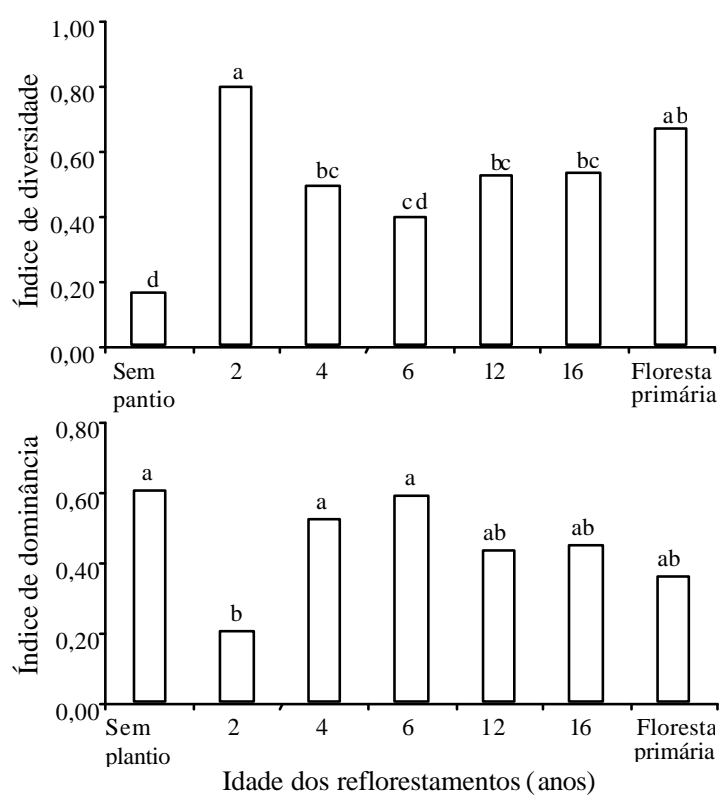

Figura 2. Índice de diversidade de espécies de fungos micorrízicos arbusculares (FMA), de Shannon-Wiener, e índice de dominância das espécies de FMA, de Simpson, das amostras de solo coletadas na área de subsolo exposto sem plantio, nas áreas revegetadas com a reposição da camada superficial orgânica há 2, 4, 6 e 12 anos, na área revegetada há 16 anos, sem a reposição da camada superficial orgânica e na floresta primária. Colunas com a mesma letra para cada índice não diferem entre si, a 5\% de probabilidade pelo teste de Mann-Witney. esporulação, indicando alta competitividade nas áreas revegetadas entre quatro e 16 anos. Na floresta primária, a presença de esporos de G. macrocarpum foi relativamente menor. Este mesmo comportamento pode ser notado na revegetação com dois anos, em que houve predomínio de espécies do gênero Acaulospora, com características de espécies pioneiras, em especial A. mellea.

\section{Conclusões}

1. O período seco propicia a esporulação de maior número de espécies de fungos micorrízicos arbusculares (FMA) que o chuvoso.

2. A produção de esporos e o número de espécies de FMA são influenciados pelo tempo da revegetação e pela reposição de solo orgânico.

3. As áreas mais perturbadas, em recuperação, produzem mais esporos de FMA do que a mata nativa em clímax, menos perturbada.

4. O retorno do horizonte superficial orgânico estimula a esporulação de FMA em subsolos estéreis resultantes da mineração de bauxita.

5. As espécies do gênero Acaulospora estão mais adaptadas ao período inicial de revegetação especialmente A. mellea.

\section{Referências}

ABBOTT, L. K.; ROBSON, A. D. Factors influencing the occurrence of vesicular-arbuscular mycorrhizas. Agriculture, Ecosystems and Environment, Amsterdam, v. 35, p. 121-150, 1991.

ALLEN, M. F. The ecology of mycorrhizae. Cambridge, England: Cambridge University Press, 1991. 184 p.

BROWER, J. E.; ZAR, J. H.; VON ENDE, C. N. Field and laboratory methods for general ecology. $3^{\text {rd }} \mathrm{ed}$. Dubuque: W. C. Brown, 1990. 237 p.

CARRENHO, R. Influência de diferentes espécies de plantas hospedeiras e fatores edáficos no desenvolvimento de fungos micorrízicos arbusculares (FMA). 1998. 226 f. Tese (Doutorado em Biologia) - Universidade Estadual Paulista, Rio Claro, 1998.

CLARK, R. B. Arbuscular mycorrhizal adaptation, spore germination, root colonization, and host plant growth and mineral acquisition at low $\mathrm{pH}$. Plant and Soil, The Hague, v. 192, p. 5-22, 1997. 
CUENCA, G.; ANDRADE, Z.; ESCALANTE, G. Diversity of glomalean spores from natural, disturbed and revegetated communities growing on nutrient-poor tropical soils. Soil Biology \& Biochemistry, Ottawa, v. 30, n. 6, p. 711-719, 1998.

DOUDS, D. D.; MILLNER, P. D. Biodiversity of arbuscular mycorrhizal fungi in agroecosystems. Agriculture, Ecosystems and Environment, Amsterdam, v. 74, p. 77-93, 1999.

FERRAZ, J. B. Soil factors influencing the reforestation on mining sites in Amazonia. In: LIETH, H.; LOHMAN, M. (Ed.). Restoration of tropical forest ecosystems. Dordrecht: Kluwer Academic, 1993. p. 47-52.

FRANKE-SNYDER, M.; DOUDS JUNIOR, D. D.; GALVEZ, L.; PHILLIPS, J. G.; WAGONER, P.; DRINKWATER, L.; MORTON, J. B. Diversity of communities of arbuscular mycorrhizal (AM) fungi present in conventional versus low-input agricultural sites in Eastern Pennsylvania, USA. Applied Soil Ecology, Amsterdam, v. 16, p. 35-48, 2001.

GERDEMANN, J. W.; NICOLSON, T. H. Spores of mycorrhizal endogone species extracted from soil by wetsieving and decanting. Transactions of the British Mycological Society, Cambridge, England, v. 46, p. 235 244, 1963.

GOULD, A. B.; HENDRIX, J. W. Relationship of mycorrhizal activity to time following reclamation of surface mine land in western Kentucky - II: mycorrhizal fungal communities. Canadian Journal of Botany, Ottawa, v. 76, p. 204-212, 1998.

GOULD, A. B.; HENDRIX, J. W.; FERRISS, R. S. Relationship of mycorrhizal activity to time following reclamation of surface mine land in Western Kentucky I: propagule and spore population densities. Canadian Journal of Botany, Ottawa, v. 74, p. 247-261, 1996.

HENDRIX, W.; GUO, B. Z.; AN, Z. Q. Divergence of mycorrhizal fungal communities in crop production systems. Plant and Soil, The Hague, v. 170, p. 131-140, 1995.

KENNEDY, A. C.; SMITH, K. L. Soil microbial diversity and the sustainability of agricultural soils. Plant and Soil, The Hague, v. 170, p. 75-86, 1995.

KIERMAN, J. M.; HENDRIX, J. W.; MARONEK, D. M. Endomycorrhizal fungi occurring on orphan strip mines in Kentucky. Canadian Journal of Botany, Ottawa, v. 61, p. 1798-1803, 1983.
KLIRONOMOS, J. N.; MOUTOGOLIS, P.; KENDRICK, B.; WIDDEN, P. A comparison of spatial heterogeneity of vesicular-arbuscular mycorrhizal fungi in two maple-forest soils. Canadian Journal of Botany, Ottawa, v. 71, p. 1472-1480, 1993.

LAPA, R. P. A bauxita e o rejeito da bauxita. In: BOZELLI, R. L.; ESTEVES, F. A.; ROLAND, F. (Ed.). Lago Batata: impacto e recuperação de um ecossistema amazônico. Rio de Janeiro: UFRJ, 2000. p. 27-35.

MARTINS, C. R.; MIRANDA, J. C. C.; MIRANDA, L. N. Contribuição de fungos micorrízicos arbusculares nativos no estabelecimento de Aristida setifolia Kinth em áreas degradadas do cerrado. Pesquisa Agropecuária Brasileira, Brasília, v. 34, n. 4, p. 665-667, abr. 1999.

MEHROTRA, V. S. Arbuscular mycorrhizal associations of plants colonizing coal mine spoil in India. Journal of Agricultural Science, Cambridge, England, v. 130, p. 125-133, 1998.

MORTON, J. B.; BENTIVENGA, S. P.; BEVER, J. D. Discovery, measurement, and interpretation of diversity in symbiotic endomycorrhizal fungi (Glomales, Zygomycetes). Canadian Journal of Botany, Ottawa, v. 73 , n. 1, p. 25-32, 1995. Supplement.

MUNYANZIZ, E.; KEHRI, H. K.; BAGYARAJ, D. J. Agricultural intensification, soil biodiversity and agroecosystem function in the tropics: the role of mycorrhiza in crops and trees. Applied Soil Ecology, Amsterdam, v. 6, p. $77-85,1997$.

NEWSHAM, K. K.; FITTER, A. H.; WATKINSON, A. R. Multifuncionality and biodiversity in arbuscular mycorrhizas. Trends in Ecology and Evolution, London, v. 10, n. 10, p. 407-411, 1995.

NOETHER, G. E. Introdução à estatística: uma abordagem não paramétrica. 2. ed. Rio de Janeiro: Guanabara Dois, $1983.258 \mathrm{p}$.

ODUM, E. P. Ecologia. São Paulo: Guanabara, 1988.330 p

PFLEGER, F. L.; STEWART, E. L.; NOYD, R. K. Role of VAM fungi in mine land revegetation. In: PFLEGER, F. L.; LINGERMAN, R. G. (Ed.). Micorrhizae and plant health. St. Paul: APS Press, 1994. p. 47-82.

PIELOU, E. C. Population and community ecology: principles and methods. New York: Gordon \& Breach, 1983. $424 \mathrm{p}$.

SALOMÃO, R. P.; ROSA, N. A.; FERRAZ, J.; MATOS, A. H. Uso de parcelas permanentes em reflorestamen-

Pesq. agropec. bras., Brasília, v. 38, n. 12, p. 1409-1418, dez. 2003 
tos de diversas idades para avaliação da recuperação de áreas mineradas, Porto Trombetas, Oriximiná, Pará. In: SIMPÓSIO BRASILEIRO DE RECUPERAÇÃO DE ÁREAS DEGRADADAS, 6., 1997, Ouro Preto. TrabaIhos voluntários. Viçosa: Sobrade, 1997. p. 407-415.

SANTOS, A. L.; SOUZA, F. A. de; BERBARA, R. L. L.; GUERRA, J. G. M. Estabelecimento e capacidade infectiva de Gigaspora margarita e Glomus clarum em solo sob erosão. Acta Botanica Brasilica, São Paulo, v. 14, n. 2, p. $127-139,2000$.

SCHENCK, N. C.; PEREZ, Y. A manual of identification of vesicular-arbuscular mycorrhizal fungi. $2^{\text {nd }} \mathrm{ed}$. Gainesville: University of Florida, 1988. 241 p.

SIEVERDING, E. Vesicular-arbuscular mycorrhiza management in tropical agrosystems. Eschborn:
Deutsche Gesellschaft für Technische Zusammenarbeit, $1991.317 \mathrm{p}$.

SIQUEIRA, J. O.; COLOZZI-FILHO, A.; OLIVEIRA, E. de. Ocorrência de micorrizas vesicular-arbusculares em agro e ecossistemas do Estado de Minas Gerais. Pesquisa Agropecuária Brasileira, Brasília, v. 24, n. 12, p. 1499 1506, dez. 1989.

TEWS, L. A.; KOSKE, R. E. Toward a sampling strategy for vesicular-arbuscular mycorrhizas. Transactions of the British Mycological Society, Cambridge, England, v. 87, p. 353-358, 1986.

WEST VIRGINIA UNIVERSITY. International culture collection of arbuscular vesicular-arbuscular mycorrhizal fungi. Disponível em: <http:// invam.caf.wvu.edu>. Acesso em: jul. 2000. 